\title{
EI Balanced Scorecard en el Desarrollo de los Negocios Familiares
}

\section{The Balanced Scorecard in the Development of Family Businesses}

Julio Cesar Méndez Bravo

Universidad de Guayaquil, Ecuador

Universidad Internacional del Ecuador, Ecuador

Haybee Jossety Ayala Briones

Universidad de Guayaquil, Ecuador

Tania Yolanda Palacios Sarmiento

Universidad Internacional del Ecuador, Ecuador

Autor corresponsal: julio.mendezb@ug.edu.ec, haybeeayala@gmail.com, tapalaciossa@uide.edu.ec Fecha de recepción: 25 de febrero del 2019 - Fecha de aceptación: 27 de agosto del 2019

Resumen: Las empresas familiares son las unidades de negocio más antiguas en el mundo empresarial, este tipo de empresas son de gran importancia dentro de un país porque dinamizan la economía y generan empleo. La subsistencia de este tipo de empresas a largo plazo es muy importante, sin embargo, gran parte de este tipo de negocios familiares llegan a fracasar antes de cumplir los 5 años de vida. Por ello es necesario el desarrollo e implementación de un sistema de gestión que logre identificar los problemas y ayude a mejorarlos, el Balanced Scorecard (BSC) es la herramienta de gestión más adecuada para solucionar problemas y ayudar a gestionar este tipo de organizaciones. El presente trabajo realizo una revisión literaria de investigaciones de alto impacto, permitiendo determinar al BSC como la mejor opción al momento de realizar la planificación y control de las actividades en las organizaciones, mejorando las expectativas de sostenibilidad y crecimiento en las organizaciones.

Palabras claves: empresas familiares; balanced scorecard; gestión; mejora continua; rentabilidad

Abstract: Family businesses are the oldest business units in the business world, these types of businesses are of great importance within a country because they boost the economy and employment results. The subsistence of this type of long-term business is very important, however, a large part of this type of family business fails before reaching the age of 5 . Therefore, it is necessary to develop and implement the network management system to identify problems and help improve, the Balanced Scorecard (BSC) is the most appropriate management tool to solve problems and help manage this type of organizations. The present work is a literary review of high impact research can be determined in the BSC as the best option at the time of planning and control of activities in organizations, improving expectations of sustainability and growth in organizations. Keywords: family businesses; balanced scorecard; management; continuous improvement; profitability 


\section{Introducción}

Las empresas familiares captan alrededor del $51 \%$ del Producto Interno Bruto y son las generadoras de empleo y mueven constantemente la economía de un país (Camino \& Bermúdez, 2018). Jaramillo (2002) en su artículo "Las empresas familiares frente a la implementación de sistemas de control" mencionó a los autores Amat (2000) y Jaramillo (1998) definieron a las empresas familiares como organizaciones privadas de negocios cuya característica principal es la transferencia de la propiedad a manos de un familiar y estas nacen en consecuencia de las necesidades propias de la familia. En estas organizaciones la familia es la fuente inicial de mano de obra. En el artículo "Empresas de familia: conceptos y modelos para su análisis", Molina, Botero \& Montoya (2016) elaboraron una tabla en la cual tomaron diferentes definiciones de lo que es una empresa familiar de diferentes autores, mencionando a este tipo de empresas como una empresa en la que una familia posee la mayoría del capital y tiene el control total, además, los miembros de la familia forman parte de la dirección e intervienen en la toma de decisiones.

Mendoza, Hernández \& Salazar (2010) citando a Ibrahim, Angelidis \& Parsa (2008) indicaron que las empresas familiares tienen características que generan mayores ventajas competitivas, las cuales están enfocadas al cliente, a la calidad y son más activas dentro de la comunidad. Las características que distinguen a las empresas familiares de las PYMES (Pequeñas y Medianas Empresas) y otras empresas no familiares son la dirección, control y gestión de la empresa (Molina, Botero, \& Montoya, 2016). Para el desarrollo del presente trabajo se consideraron trabajos e investigaciones referente a las PYMES y las empresas familiares. Considerando que existen diferentes definiciones de empresa familiar, autores como Camino \& Bermúdez (2018) y Vallejo (2005) los cuales concluyeron que, a pesar de múltiples aportaciones, la definición del término empresa familiar aun no es unánime ya que su definición depende de ciertos factores que la caractericen como empresa familiar. Bañegil, Barroso y Tato (2011) indicaron que la empresa familiar es una organización social de gran complejidad, donde conviven la empresa y la familia. Operativamente, de acuerdo con Lozano (2000), la empresa familiar también puede definirse como un tipo de unidad empresarial que suele operar a partir de la disponibilidad de capital y trabajo de origen familiar o doméstico. Por consiguiente, para el presente trabajo se definirá a la empresa familiar como la unidad económica básica generadora de desarrollo económico y social, basados en capital, recursos y administración familiar.

Las grandes empresas surgen de negocios familiares, en la actualidad la competencia es cada vez más fuerte y las exigencias de los clientes es creciente. Basados en el trabajo de Camino \& Bermúdez (2018) titulado Empresas familiares en el Ecuador: Definición y aplicación metodológica, se puede establecer que en el Ecuador la presencia de empresas familiares tiene como visión común: ser exitosos y rentables, ya que de ello dependerá el futuro y el crecimiento económico de la familia. Basados en la información previa, el presente trabajo plantea la siguiente interrogante: “¿Cómo los negocios familiares pueden llegar al éxito mediante la implementación de BSC?"; si bien es cierto las empresas familiares son emprendedores, pero este desconocimiento es el principal problema del fracaso en los negocios y los emprendimientos, entre los cuales se puede presentar la inadecuada ejecución y gestión de las empresas (Sánchez \& Araujo, 2016). 
Las empresas familiares y no familiares deben saber que en este tiempo (era de la información) el éxito de las empresas se basa en la calidad de la organización mediante la satisfacción de las necesidades de los clientes respecto a sus exigencias y requerimientos. El BSC permite conocer de una forma integrada, balanceada y estratégica, el desempeño de una organización mediante un conjunto coherente de indicadores agrupados en cuatro perspectivas, lo que permite ver a la organización en su conjunto (Martínez, 2011). Esta herramienta metodológica fue desarrollada por Kaplan y Norton a principios de los años 90 siendo una herramienta utilizada a lo largo de los últimos años por muchas organizaciones (Sánchez \& Araujo, 2016). El BSC no se limita al tamaño de la empresa ni a su sector de actividad o etapa de ciclo de vida. El BSC es una herramienta de gestión que ayuda a una empresa a cumplir su visión, a través de la ejecución de objetivos estratégicos los cuales están enfocados principalmente a la generación de valor (Quintero \& Osorio, 2018). El BSC identifica indicadores financieros y no financieros que ayudaran a formular estrategias para alcanzar la visión propuesta por la empresa.

Arias, Castaño \& Lanzas (2005) mencionaron que el BSC es una herramienta que tiene como objetivo, desarrollar estrategias de cualquier tipo de empresa u organización en forma integral, balanceada y estratégica. Esta herramienta permite el direccionamiento de una organización de forma proactiva (Montoya \& Bárbaro, 2011).

\section{La Empresa Familiar}

La empresa familiar es la estructura productiva más antigua de la humanidad, representando un rol importante en la economía del país (Camino \& Bermúdez, 2018). Basco (2006) mencionó al autor Colli (2003) quien indicó que las empresas familiares han tenido un espacio sobresaliente antes y durante la revolución industrial. La característica de estas empresas es que los miembros de la organización comparten la sangre, trabajo y propiedad y que la mano de obra principal es la familia. Para Goyzueía (2013) las empresas familiares son esenciales para el desarrollo económico de un país, por lo que deberían de incrementar su actividad económica y comunicación empresarial, lo que les permitirá subsistir y ser sostenibles en el tiempo.

En este artículo se tomaron las definiciones dadas por los autores Grabinsky \& Steider (2000) que la definieron como la empresa cuya propiedad, dirección y control de las operaciones está en manos de una familia siendo ellos quienes toman las decisiones básicas estratégicas y operativas y asumen por completo la responsabilidad de sus acciones y Vélez (2008) quien mencionó que es aquella en cual las personas de una o más familias ligadas por vínculos de parentesco poseen suficiente propiedad accionaria de la empresa, el control de decisiones y aplican un modelo de gestión interdependiente con el proceso de sucesión. Mundialmente las empresas familiares representan más de dos tercios de todas las compañías existentes, convirtiéndose en pilares fundamentales en el desarrollo económico y social de los países y siendo estas las que generan empleo (Camino \& Bermúdez, 2018).

Una característica interesante de estas empresas es que tienen más partes interesadas y mayor número de interrelaciones entre ellas, los accionistas de las empresas familiares tienen un compromiso distinto con la empresa y los ciclos de evolución y el desarrollo de la empresa cambian en base a las necesidades de la familia (Molina, Botero, \& Montoya, 2016). En este tipo

Esta obra se comparte bajo la licencia Creative Common Atribución-No Comercial 4.0 International (CC BY-NC 4.0) 
de organizaciones hay una relación directa entre la empresa y la familia es decir si algo afecta a la empresa también afectara a la familia y viceversa. La influencia de la familia en estas empresas mayormente en Latinoamérica es muy importante (Goyzueía, 2013).

Es importante conocer que se puede confundir a la empresa familiar con una PYMES, sin embargo, ambos términos pueden ser relacionados, pero no considerarlos como iguales. Las distinciones que separan estos tipos de organizaciones con otras es la manera en la que formulan y ejecutan la estrategia (Molina, Botero, \& Montoya, 2016), una característica que logra diferenciarlas es como está formada y llevada la dirección, control y gestión de la misma. Al ser la empresa familiar una PYMES se la considera como la especie empresarial en el mundo más ágil y más capaz de competir en el actual entorno cambiante (era de la información) y capaz de adaptarse a cambios en su entorno. Una empresa familiar puede ser una PYMES, pero no toda PYMES puede ser una empresa familiar.

Aíra (2016) mencionó que la empresa familiar es aún más rentable que las empresas no familiares por el nivel de compromiso y rapidez en la toma de decisión, este tipo de empresas son tan importantes porque mueven constantemente la economía del país y son tomadas como patrimonio en el núcleo familiar, la empresa familiar se destaca porque quienes crearon la empresa tienen una visión a largo plazo, ya que su legado es para las generaciones futuras con el fin de seguir generando beneficios. Para Aíra (2016) en relación a Goyzueía (2013) la empresa familiar se destaca por su capacidad de mantener su crecimiento a largo plazo y por haber superado problemas como la sucesión, rivalidad y falta de profesionalismo. Como se puede apreciar la necesidad de mantenerse y generar una sostenibilidad en el mercado ha obligado a la evolución de las empresas familiares.

En Ecuador la mayoría de empresas son de propiedad familiar, no hay que limitar el tamaño de la empresa familiar ya que esta puede ser pequeña o grande incluso las grandes organizaciones nacieron como empresas familiares tales como Walmart. Camino \& Bermúdez (2018) mencionaron que "En Ecuador, según un estudio de la Empresa Familiar de Ecuador realizado por la Universidad de Especialidades Espíritu Santo (UEES), el 90,5\% de las empresas ecuatorianas registradas en la Superintendencia de Compañías, Valores y Seguros tienen una estructura de tipo familiar. Un 7,9\% de las empresas son de propiedad dispersa no familiar y el 1,7\% de propiedad desconocida. Adicionalmente, del conjunto de grandes empresas, considerando las empresas que poseen una nómina igual o superior a los 250 empleados, el $65,9 \%$ son de propiedad familiar, mientras que, en el segmento de empresas medianas este tipo de estructura representa un $88 \%$, finalmente, las empresas pequeñas y microempresas representan más del $90 \%$ como empresas familiares" p. 48. Se puede establecer que el impacto que tiene este tipo de empresa en el Ecuador es relevante en su desarrollo económico, razón por la cual se hace necesario la implementación de una herramienta que permita su sostenibilidad y crecimiento.

A continuación, en la Tabla 1 se pueden apreciar investigaciones de empresas familiares y del BSC de forma separada, así como investigaciones que presentan al BSC como una herramienta favorable para el desarrollo de las actividades de las empresas familiares. 
Tabla 1: Análisis del Balanced Scorecard y Las empresas familiares

\begin{tabular}{llll}
\hline \multicolumn{1}{c}{ Autor } & Paper & Objetivo del Paper & Conclusión \\
\hline \multicolumn{1}{c}{ Camino, } & Empresas Familiares & $\begin{array}{l}\text { Construir una metodología que } \\
\text { permita diferenciar a las empresas }\end{array}$ & $\begin{array}{l}\text { Las empresas ecuatorianas se } \\
\text { adecuan a la definición propuesta }\end{array}$ \\
Bermúdez, & en el Ecuador: & Definición y aplicación & $\begin{array}{l}\text { según su estructura de propiedad. } \\
\text { demostrando que la mayoría de }\end{array}$ \\
N. (2018) & metodológica. $X-$ & Aportar en la literatura la & empresas en el Ecuador son de tipo \\
& $\begin{array}{l}\text { Pendientes Económicos, } \\
\text { vol. 2(3), pp. 46-72 }\end{array}$ & y no famión de una empresa familiar y sus diferencias & familiar
\end{tabular}

Quintero, Balanced Scorecard

L. \&

Osorio, L. (2018) como herramienta para las empresas en estado de crisis. Revista CEA, vol.4 (8), pp. 75-94

Proaño, Mejora continua H., Gisbert, enfocada a los V. \& Pérez, problemas de empresas E. (2017) familiares. 3C Empresa, investigación y pensamiento crítico, Edición Especial, pp. 29-38

Suárez, F. Análisis de la \& aplicación del Balanced Gonzáles, Scorecard Como M. (2017) herramienta de diagnóstico adaptada a microempresa. Revista de economía \& administración, vol. 14(1), pp. 99-110

Aira, M. La sucesion exitosa en (2016) las empresas Familiares. Orbis.Revista Cientifica Electronica de Ciencias Humanas, vol.11(33), pp. $82-104$

Quejada, R. \& Ávila, J. (2016)

Empresas familiares: conceptos, teorias y estructuras. Revista de
Analizar la pertinencia del BSC en empresas en crisis, mediante una exploración documental que posibilite comprender las oportunidades que brinda esta herramienta.

Identificar los principales problemas que existen en las empresas familiares y cuáles son las que dificultan la solución de problemas, el artículo pretende mejorar la gestión de estas empresas.

Desarrollar un análisis de la aplicación del BSC como herramienta para el diagnóstico de las microempresas a fin de que estas unidades productivas puedan contar con los beneficios de esta metodología

Establecer las características requeridas para la sucesión exitosa en empresas familiares del Estado Carabobo. Escuela de Administracion de Negocios,(81), pp. 149158

Presenta aspectos conceptuales, teóricos, históricos y estructurales, de las empresas familiares. Se destaca el rol que desempeñan en materia de generación de empleo y riqueza.
El BSC es una herramienta de gran utilidad y su implementación tiene un efecto positivo y necesario dentro de las organizaciones en crisis.

Los lazos que comparten las empresas familiares dificultan la toma de acciones y reclamaciones en cuanto a la gestión y administración de ellas, se presenta al BSC para mejorar la organización y optimizar sus procesos y gestión

El BSC permitió identificar las necesidades y los principales problemas de estas unidades. Es muy flexible y tiene una capacidad de adaptación que brinda un panorama a la hora de medir el desempeño de la organización, permite al emprendedor un abanico de usos de esta herramienta.

Con una adecuada definición de las empresas familiares, sus características, señalo que la sucesión exitosa de estas empresas tiene relación con la parte afectiva de ellos además de características que deben tener los miembros de la familia.
Las EF son motores de valor agregado y crecimiento, sin embargo, la debilidad de la sucesión puede llegar a afectar su característica de contribuir a la dinamización y sostenibilidad de las ellas. 
Autor Paper

Ruiz, F., $\quad$ Concepto de familia
Saldaña, Y., $\quad$ en la cultura mexicana y

Gaona, L. su impacto en la

\& Jacobo, empresa familiar.

D. (2016) Revista Internacional

Administración y

Finanzas, vol.9 (5), pp.

$15-25$

Sánchez, Balanced Scorecard

J. \& Araújo, para emprendedores:

P. (2016) Desde el modelo canvas

al Cuadro de Mando

Integral. Revista de la

facultad de ciencias

economicas de la

Universidad Militar de

Nueva Granada,

XXIV(1), pp. 37-47

Amat, O., La implementación del Banchieri, cuadro de mando L. \& integral en el sector

Campa-

Planas, F.

(2016)

agroalimentario: El caso

del grupo alimentario

Guissona. Revista de la

facultad de ciencias

económicas, vol.24 (1), pp. 25-36

Goyzueía, Modelo de gestión

S. (2013) para las empresas con

perspectivas de

crecimiento y

sostenibilidad.

Perspectivas,

vol.16(31), pp. 87-132

Montoya, The Balanced

C. \& Scorecard as a control

Bárbaro, J. system and its

(2011) application as

evaluation tool in the

library environment.

Revista Interoamericana

de Bibliotecnología,

vol.34 (1), pp. 35-47

Objetivo del Paper

Conclusión

Mostrar cómo a pesar de los cambios en las normas sociales que regulan el comportamiento del individuo, el concepto de familia aún está enraizado en la cultura del mexicano y explica cómo dicho concepto puede impactar la dinámica y continuidad de la empresa familiar

Desarrollar un modelo que parte de la necesidad de disponer del BSC y relacionar ese diseño con el modelo Canvas señalando su mutua necesidad.

Determinar la importancia de la implantación de un Cuadro de Mando Integral (CMI) para poder monitorear la referida estrategia.

Abordar la dinámica de la empresa familiar y de esta manera poder presentar un modelo de gestión que asegure el crecimiento, permanencia y continuidad de este tipo de empresas.

Analizar su aplicación en el ámbito bibliotecario desde sus cuatro perspectivas: cliente, financiera, procesos internos y aprendizaje, y crecimiento. Con el fin de demostrar su utilidad en las unidades de información y la construcción de modelos de gestión.
A pesar de los cambios sociales ocurridos, la red familiar es aun considerablemente extensa y las relaciones se caracterizan por ser continuas a través del tiempo. Para el autor las decisiones tienen un gran impacto y pueden afectar la rentabilidad y continuidad del negocio.

Los emprendedores deben tomar en cuenta y usar el BSC junto con el modelo Canvas porque estas dos herramientas permiten poner en marcha el negocio y reducir el riesgo de fracaso en los primeros años de vida de la empresa.

La implementación del BSC es un proyecto que tiene viabilidad en esta organización. El autor menciona que en caso de implementar el BSC se debería estudiar los resultados obtenidos del mismo.

Las empresas familiares son muy importantes en la economía del país sin embargo, presentan

inconvenientes que las pueden llevar al fracaso, por lo cual se diseñó un modelo de gestión para este tipo de empresas.

El BSC es un instrumento práctico y útil para las unidades de información; no es la solución a todos sus puntos débiles, pero brinda la posibilidad de mejorar los aspectos estratégicos al establecer las relaciones causa-efecto entre los objetivos y los indicadores en sus cuatro perspectivas. 


\begin{tabular}{|c|c|c|c|}
\hline Autor & Paper & Objetivo del Paper & Conclusión \\
\hline $\begin{array}{l}\text { Sánchez, } \\
\text { F. (2010) }\end{array}$ & $\begin{array}{l}\text { El Balanced Scorecard } \\
\text { como herramienta de } \\
\text { gestión en las } \\
\text { organizaciones del siglo } \\
\text { XXI. Gestión y } \\
\text { desarrollo de la } \\
\text { Universidad de } \\
\text { Buenaventura-Cali, Vol. } \\
\text { 7(2), pp. 123-132 }\end{array}$ & $\begin{array}{l}\text { Exponer brevemente la } \\
\text { importancia del Balanced } \\
\text { Scorecard como herramienta de } \\
\text { gestión y modelo de planificación } \\
\text { que permite alinear la organización } \\
\text { con su estrategia. }\end{array}$ & $\begin{array}{l}\text { El BSC puede ayudar a planificar, } \\
\text { entender y comunicar mejor las } \\
\text { estrategias organizacionales, y a } \\
\text { gestionar la visión más globalmente } \\
\text { y a largo plazo. }\end{array}$ \\
\hline $\begin{array}{l}\text { Esparza, } \\
\text { J., García, } \\
\text { D. \& } \\
\text { Duréndez, } \\
\text { A. (2009) }\end{array}$ & $\begin{array}{l}\quad \text { Gestión estratégica y } \\
\text { competitiva de las } \\
\text { empresas familiares } \\
\text { turísticas mexicanas: Un } \\
\text { estudio empírico. } \\
\text { Revista EAN, (66), pp. } \\
\text { 5-30 }\end{array}$ & $\begin{array}{l}\text { Analizar las principales } \\
\text { diferencias de la gestión } \\
\text { estratégica y competitiva de las } \\
\text { micro, pequeñas y medianas } \\
\text { empresas (Mi Pyme) familiares y } \\
\text { no familiares }\end{array}$ & $\begin{array}{l}\text { Las Empresas Familiares hacen } \\
\text { uso de diferentes factores de } \\
\text { competitividad y herramientas de } \\
\text { gestión se encontraron con algunas } \\
\text { debilidades que Empresas Familiares } \\
\text { deberían subsanar. }\end{array}$ \\
\hline $\begin{array}{l}\text { Cordova, } \\
\text { L. (2008) }\end{array}$ & $\begin{array}{l}\text { Aplicación del } \\
\text { Balanced Scorecard } \\
\text { como metodología de } \\
\text { gestión en las MYPES } \\
\text { Y PYMES peruanas. } \\
\text { Ingeniería Industrial, } \\
\text { (26), pp. 85-97 }\end{array}$ & $\begin{array}{l}\text { Identificar al Balanced Scorecard } \\
\text { como herramienta de gestión en las } \\
\text { MI Pymes siendo esta que ayuda a } \\
\text { enfrentar con éxito el entorno } \\
\text { competitivo. }\end{array}$ & $\begin{array}{l}\text { Se construye el modelo de gestión } \\
\text { para estas empresas la cual contiene } \\
\text { la relación y análisis de las } 4 \\
\text { perspectivas del BSD, se definen los } \\
\text { objetivos y los indicadores que } \\
\text { ayudaran a llegar a ellos y por } \\
\text { consiguiente el autor revelo los } \\
\text { resultados de cada indicador. }\end{array}$ \\
\hline $\begin{array}{l}\text { Craig, J. } \\
\& \text { Moores, } \\
\text { K. (2005) }\end{array}$ & $\begin{array}{l}\quad \text { Balanced Scorecards } \\
\text { to drive the strategic } \\
\text { planning of family } \\
\text { Business (Esparza, } \\
\text { García, \& Duréndez, } \\
\text { 2009). Family Business } \\
\text { Review, vol.18 (2), pp. } \\
\text { 105-122 }\end{array}$ & $\begin{array}{l}\text { La herramienta de medición y } \\
\text { gestión conocida como Balanced } \\
\text { Scorecard (BSC) y cómo se puede } \\
\text { aplicar en el contexto de la } \\
\text { empresa familiar. }\end{array}$ & $\begin{array}{l}\text { La escala F-PEC proporciona } \\
\text { cómo las empresas familiares } \\
\text { pueden profesionalizar su gestión. }\end{array}$ \\
\hline
\end{tabular}

\section{El Balanced Scorecard}

El BSC es una herramienta diseñada por Kaplan y Norton a principios de los años 90 siendo una herramienta utilizada a lo largo de los últimos años por diferentes organizaciones (Sánchez \& Araujo, 2016). Montoya \& Bárbaro (2011) citando a Torres (2002), indicó que el trabajo de los profesores fue un gran aporte a la gestión organizacional de las empresas. El BSC se da a conocer como una herramienta de gestión estratégica que permite tener bajo control y relacionadas todas aquellas medidas que representan las variables claves para dirigir un negocio. Identifica indicadores financieros y no financieros que ayudaran a formular estrategias para alcanzar la visión propuesta por la empresa.

El BSC es un modelo de gestión que ayuda a una empresa a cumplir su visión, a través de la ejecución de unos objetivos, los cuales están enfocados principalmente a la generación de 
valor (Quintero \& Osorio, 2018). También se puede indicar que proporciona mecanismos necesarios para orientar a la organización hacia su estrategia (Arias, Castaño, \& Lanzas, 2005). En la investigación desarrollada por Kaplan y Norton en 12 empresas, evidenciaron que dichas empresas solo se fijaban en indicadores financieros como medida de desempeño de la organización y que en base a ella formulaban las estrategias y tomaban las decisiones, el BSC menciona que los indicadores financieros sirven para medir las actividades efectuadas en esa área y que son complementadas por otras medidas de desempeño para la organización. Por ello el BSC no busca reemplazar las mediciones tradicionales sino complementarlas (Arias, Castaño, \& Lanzas, 2005).

En esta nueva era (era de la información) los administradores comprenden que los activos tangibles e intangibles son factores que permiten que una organización desarrolle diversas características que le permitirán generar un mayor nivel de competencia y estabilidad en el mercado (Quintero \& Osorio, 2018). La implementación del BSC moviliza al personal de las organizaciones hacia el pleno conocimiento de la misión, canalizando para ello sus energías, habilidades y conocimiento específico en la organización hacia el logro de metas estratégicas de largo plazo, permite tanto guiar el desempeño actual como apuntar al desempeño futuro (Sánchez, 2010).

El BSC permite el direccionamiento de una organización de forma proactiva y esta no se limita al tipo de empresa, tamaño o propósito (Montoya \& Bárbaro, 2011). El BSC se lo ha aplicado en diferentes tipos de organizaciones, sea en su tamaño, gestión, propósito y estado (rentable o en crisis). Al aplicar el BSC se logra simplificar la gestión, priorizar lo importante, alinear la organización y promover la importancia sobre ellos, además de una mejora exhaustiva dentro de la misma, un personal comprometido y eficiente (Sánchez, 2010). Según Fernández (2001) el BSC permite identificar las actividades y recursos ineficientes dentro de la organización, obligando a una reestructuración, con el fin de mejorar sustancialmente los procesos y llegar a la mejora continua.

El BSC mide el desempeño a través de indicadores que se establecen a partir de cuatro perspectivas: financiero, cliente, procesos internos y aprendizaje y crecimiento, estas cuatro perspectivas interactúan entre sí y con el diseño de objetivos cooperan para cumplir con el objetivo general que se haya planteado la organización. Por lo tanto, el BSC permite ver a la organización como un todo (Sánchez \& Araujo, 2016).

La importancia del BSC radica en que es una herramienta que favorece a la administración de las empresas, mejora los procesos, alinea al personal al logro de la visión permitiendo trabajar como un solo ente donde todos sus elementos aportan al crecimiento y cumplimiento de metas planteadas. Muchas organizaciones han afirmado el éxito que se han propuesto como organización, lo han alcanzado debido a la implementación de esta herramienta (Quintero \& Osorio, 2018).

\section{Las Empresa Familiar y su Relación con el BSC}

Goyzueía (2013) así como Santamaría \& Pico (2015) mencionaron que muchos creen que las empresas familiares logran mantener el éxito a largo plazo sin embargo hay otros autores

Esta obra se comparte bajo la licencia Creative Common Atribución-No Comercial 4.0 International (CC BY-NC 4.0) Revista de la Universidad Internacional del Ecuador. URL: https://www.uide.edu.ec/ 
como Rodríguez (1997), Valda (2012), Gavica y Garay (2012) mencionaron que estas organizaciones no logran subsistir a largo plazo debido a que afrontan dificultades como la sucesión, carencia de un adecuado sistema administrativo, operaciones con sistemas inadecuados, manejo ineficiente de los recursos y el control de la familia. Basados en lo antes citado, se puede establecer que las empresas familiares deben de buscar subsistir y ser sostenibles en el tiempo, ya que son un factor determinante en el desarrollo social y económico de un país. La implementación del BSC como herramienta de gestión, permitirá dicho sostenimiento.

$\mathrm{Al}$ analizar la relación entre las empresas familiares y el BSC se puede establecer que el BSC al ser una herramienta de gestión que permite tener bajo control y relacionadas todas aquellas medidas que representan las variables claves para dirigir un negocio; que permite direccionar la empresa de forma proactiva y moviliza a la gente al pleno logro de la misión y alcanzar la visión de la empresa, el BSC se debe convertir en la herramienta adecuada para ayudar y mejorar las organizaciones de tipo familiar permitiendo alcanzar el éxito y solucionar los problemas adyacentes (Kaplan \& Norton, 2011).

Altair (2005) mencionó que el presente y el futuro inmediato del BSC es el convertirse en una herramienta clave para la gestión del cambio estratégico en las organizaciones, un nuevo instrumento de gestión empresarial que permita adaptarse rápidamente a los frecuentes cambios de dirección estratégica causados por un entorno competitivo cada vez más demandante.

De acuerdo con Hudson, Smart y Bourne (2001) una de las ventajas que poseen las empresas familiares pequeñas al momento de implementar el BSC es que su estructura plana y menos burocrática le permite reaccionar más rápidamente a los cambios, que las empresas grandes. Adicionalmente, Andersen, Cobbold y Lawrie (2001) indicaron que este tipo de empresas tienen un mayor grado de flexibilidad e innovación frente a las demás organizaciones. Por su parte Von Bergen y Benco (2004) mencionaron que las pequeñas empresas familiares requieren una cantidad minina de información para poder implementar el BSC y poder medir su desempeño.

Por consiguiente, la eficiente implementación del BSC en una empresa familiar estará condicionada a la adecuada implementación por parte de quienes la dirigen, lo que hace necesario entender la relevancia de lo que se desea medir según cada perspectiva. Por ello a continuación muestra en la Tabla 2 las preguntas que deben de responder en cada perspectiva según la conclusión de Sánchez (2010).

Tabla 2: Preguntas y Enfoques Estratégicos por Perspectivas

\begin{tabular}{clc}
\hline Perspectiva & Pregunta & Enfoque estratégico \\
\hline $\begin{array}{c}\text { Perspectiva } \\
\text { Financiera }\end{array}$ & $\begin{array}{c}\text { ¿Cómo nos vemos a } \\
\text { los ojos de los }\end{array}$ & $\begin{array}{c}\text { Análisis de las estrategias de crecimiento, rentabilidad y } \\
\text { riesgo, vistas desde la perspectiva del accionista }\end{array}$ \\
$\begin{array}{c}\text { Perspectiva } \\
\text { Cliente }\end{array}$ & $\begin{array}{c}\text { ¿Cómo nos ven los } \\
\text { clientes? }\end{array}$ & $\begin{array}{c}\text { La estrategia para crear valor, diferenciación y como cubre la } \\
\text { demanda desde la perspectiva del cliente. }\end{array}$ \\
Perspectiva & ¿En qué debemos & Análisis estratégico de los distintos procesos del negocio que \\
Procesos Internos & sobresalir? & crean satisfacción para el cliente y los accionistas
\end{tabular}




\begin{tabular}{cll}
\hline Perspectiva & Pregunta & Enfoque estratégico \\
\hline $\begin{array}{l}\text { Perspectiva } \\
\text { Desarrollo y }\end{array}$ & $\begin{array}{c}\text { ¿Podemos continuar } \\
\text { mejorando y creando }\end{array}$ & $\begin{array}{c}\text { Estrategias para crear un clima que soporte el cambio, la } \\
\text { innovación y el crecimiento organizacional, con una buena } \\
\text { comunicación y recurso humano. }\end{array}$ \\
\hline
\end{tabular}

Nota: Tabla elaborada mediante la adaptación de Sánchez (2010) y Montoya, Castaño \& Lanzas (2005)

Las preguntas y el enfoque estratégico de las perspectivas del BSC proporcionan un panorama claro sobre lo que se debe realizar en cada perspectiva, con ello es importante considerar los siguientes puntos que lograran la implementación eficiente del BSC:

- Se debe de definir de forma clara y precisa la misión, visión y valores según las necesidades y realidad de la organización.

- Tener claro el contexto actual de la organización, sus aspectos internos y externos (Peñaherrera, Ortiz, \& Herrera, 2017).

- Definir los objetivos estratégicos según cada perspectiva, sin olvidar el objetivo general de la organización.

- Establecer las estrategias acordes al planteamiento de los objetivos estratégicos de cada perspectiva.

- Construcción del mapa estratégico para poder visualizar como se vinculan los objetivos estratégicos.

- Determinar indicadores que permitan medir el cumplimiento de los objetivos.

- Construir programas e iniciativas, parten de los objetivos estratégicos.

- Asignar responsabilidades y recursos para el cumplimiento de los objetivos.

- Desarrollar una hoja de ruta donde se establezcan las acciones de mejora en caso que no se cumplan los indicadores.

La Figura 1 presenta un ejemplo de mapa estratégico y resume la relación que existe entre las perspectivas con sus respectivos objetivos estratégicos y con sus indicadores las flechas verticales muestran como los objetivos estratégicos pese a que pertenecen a diferentes perspectivas, muestran la relación que tienen entre sí, ya que el cumplimiento de un objetivo estratégico de una perspectiva, puede depender del cumplimiento de otro objetivo estratégico de una perspectiva diferente; de la misma forma las flechas horizontales muestran la relación de las perspectivas con sus respectivos objetivos estratégicos y con sus indicadores. Es interesante observar como el desarrollo del mapa estratégico resume todas las actividades desarrolladas y mostrar la interacción que se generan entre perspectivas.

El BSC ayudará al desarrollo de las tareas para alcanzar los objetivos y metas seguidamente en la gestión ayudará a simplificar todo y priorizar lo importante, maximizar los recursos y optimizar los tiempos de los procesos además permitirá canalizar las habilidades del personal de la organización hacia el cumplimiento de las tareas dentro las estrategias planteadas por cada perspectiva del BSC (Belloso, Ramón, \& Flórez, 2016). 


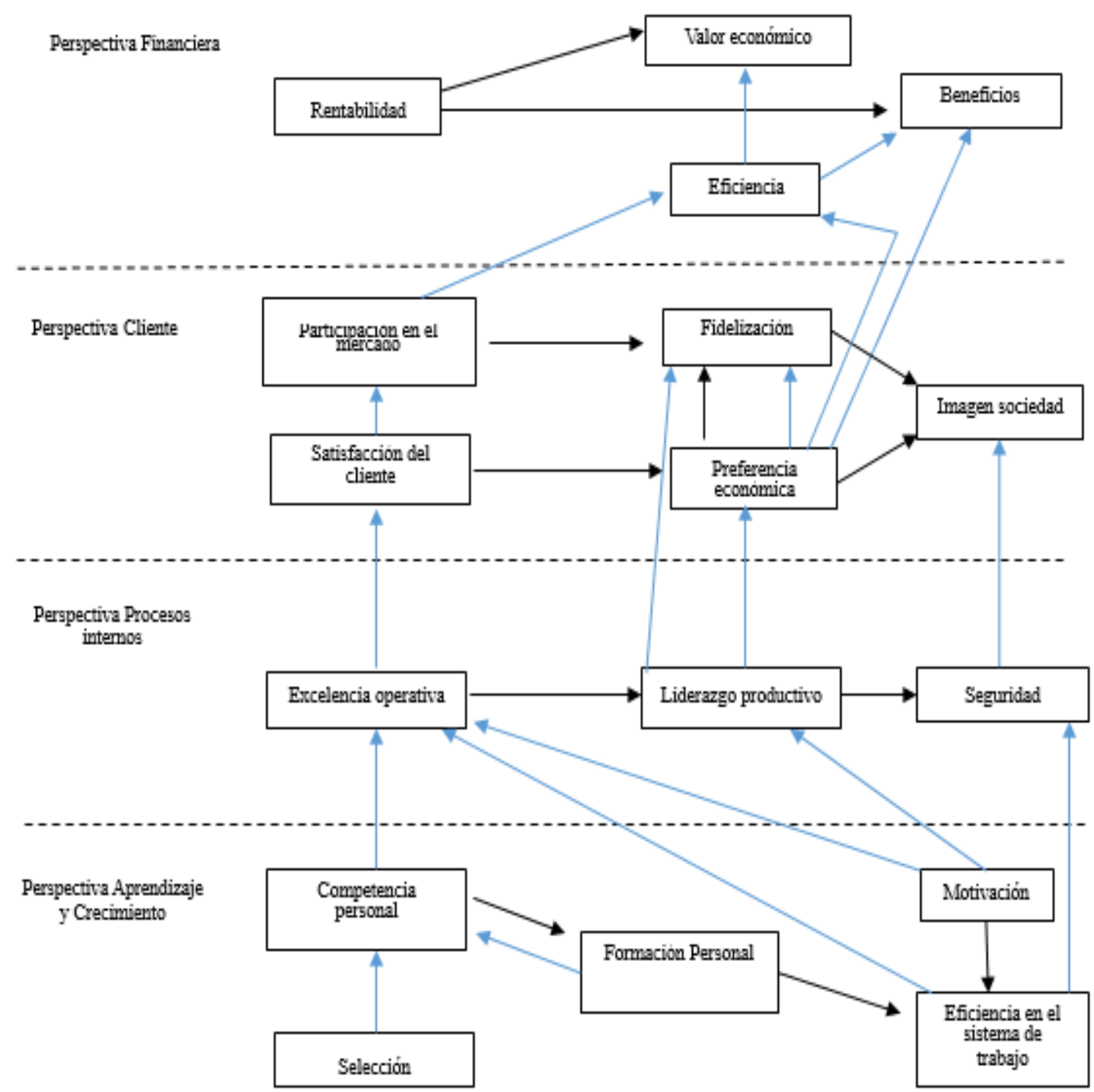

Figura 1. Mapa Estratégico. Relación Objetivos Estratégicos e Indicadores

\section{Metodología de la Investigación}

En el desarrollo del presente artículo se empleó la metodología de la revisión literaria, la cual consiste en extraer y recopilar la información relevante sobre variables a analizar desde diferentes perspectivas para generar nuevo conocimiento (Cortés \& León, 2004). Para la presente investigación se empleó papers de revistas de alto impacto publicadas entre 1992 y 2018 . La búsqueda bibliográfica se realizó a través de buscadores y base de datos académicas multidisciplinarias de algunas universidades y de revistas. Se tomaron como referencias artículos y libros referentes a los temas a discutir como son la empresa familiar, el BSC y gestión, además se debe mencionar que se utilizó como guía metodológica la investigación desarrollada por Dueñas, Perdomo, \& Villa (2014). 


\section{Conclusiones}

Basado en la revisión literaria, el BSC es una herramienta que es utilizada para la gestión, medición y control en las organizaciones, sin importar el tamaño, tipo u oficio. Permite a las organizaciones equilibrar y gestionar todas las partes que conforman la organización, direccionando a todos al cumplimiento de la visión de la misma. Como menciono Scaramussa (2010), el BSC es un sistema completo de gerencia que permite la integración tanto de aspectos del direccionamiento estratégico, como la misma evaluación de desempeño que ha tenido el negocio. Se puede concluir que el BSC es la herramienta ideal para ayudar al desarrollo del buen desempeño y sostenibilidad en las organizaciones, puede ejercer el mismo impacto en las empresas familiares. El aporte que brinda la presente investigación mediante la revisión literaria, es evidenciar y sugerir la implementación del BSC como la herramienta de gestión que permitirá de gestionar de forma eficiente el cumplimiento de los objetivos estratégicos que se planteen las empresas familiares, sobre todo al evidenciar con datos que las empresas familiares son las organizaciones que tienden a permitir el desarrollo económico y crecimiento de un país, lo que hace que su subsistencia en los mercados sea trascendental.

Al considerar lo expuesto por Montoya (2011), donde menciono que el BSC es un sistema de gestión que requiere del compromiso y la participación de todos los servidores para alcanzar el éxito y los beneficios esperados en la organización. El BSC permitirá a las empresas familiares ser competitivas en una era donde el flujo de información y eficiencia en los procesos es la diferencia entre una empresa posicionada en el mercado y uno empresa que tiende a ser desplazada. La calidad y la satisfacción del cliente se verán reflejada en la mejora sostenida de los beneficios de las empresas. El BSC permitirá una mejor forma de planificar y gestionar los negocios en las Empresas Familiares, permitiéndoles enfocar en la creación de valor, y al cumplimiento de la visión de la organización.

Para investigaciones futuras se recomienda realizar un análisis entre empresas familiares de diferentes sectores para comprobar la aplicación del BSC en ese tipo de empresas, se plantea la interrogante ¿Cómo varia la implementación del BSC entre empresas Familiares de diferentes sectores productivos?

\section{Bibliografía}

Aira, M. (2016). La sucesión exitosa en las empresas familiares. Orbis. Revista Científica Ciencias Humanas, 11(33), 82-104.

Almanzán, J., Méndez, A., \& Lee, H. (2013). Balanced Scorecard (BSC). ¿Una herramienta innovativa para las pymes mexicanas? INCEPTUM, 8(15), 85-109.

ALTAIR CONSULTORES, S.R.L., (2005). Cuadro de mando integral. Revista Economía, 3 (150), España.

Amat, J. (2000). La continuidad de la Empresa Familiar (2da ed.). Barcelona, España: Gestión 2000.

Amat, O., Banchieri, L., \& Campa, F. (2016). La implementación del Cuadro de Mando Integral en el sector agroalimentario: El caso del grupo alimentario Guissona. Revista de la Facultad de Ciencias Económicas, 24(1), 25-36. 
Andersen, H., Cobbold, I. y Lawrie, G. (2001). Balanced scorecard implementation in SMEs: reflection on literature and practice. Trabajo presentado en SME Conference, Copenhage. Recuperado de http://citeseerx.ist.psu.edu/viewdoc/download?doi=10.1.1.183.8335\&rep=rep1\&type=pdf

Arias, L., Castaño, J., \& Lanzas, A. (2005). Balanced Scorecard en Instituciones de Educación Superior. Scienta et Technica, 27, 181-184.

Bañegil, T., Barroso, A. y Tato, J. (2011). Profesionalizarse, emprender y aliarse para que la empresa familiar continúe. Revista de Empresa Familiar, 1(2), 27-41

Basco, R. (2006). La investigación en la empresa familiar: "Un debate sobre la existencia de un campo independiente". Investigaciones Europeas de Dirección y Economía del país, Vol. 12(1), 33-54.

Belloso, R., Ramón, J., \& Flórez, R. (2016). Diseño de Cuadro de Mando Integral en escuelas aeronáuticas. Revista Iberoamerica de Contabilidad de Gestión, 14(28), 1-38.

Camino, S., \& Bermúdez, N. (2018). Empresas familiares en el Ecuador: Definición y aplicación metodológica. X-Pendientes Económicos, 2(3), 46-72.

Córdova, L. (2008). Aplicación del Balanced Scorecard como metodología de gestión en las MYPES y PYMES peruanas. Ingeniería Industrial, 26, 85-97.

Craig, J., \& Moores, K. (2005). Balanced Scorecard to drive the strategic planning of family business. Family Businees Review, 18 (2), 105-122.

Dueñas, S., Perdomo, J., \& Villa, L. (2014). El concepto de consumo socialmente responsable y su medición. Una revisión de la literatura. Estudios Gerenciales, 30, 287-300.

Esparza, J., García, D., \& Duréndez, A. (2009). Gestión estratégica y competitiva de las empresas familiares turísticas mexicanas: Un estudio empírico. Revista EAN, 66, 5-30.

Fernández, A. (2001). El Balanced Scorecard: ayudando a implantar la estrategia. Revista de Antiguos Alumnos, 31-44.

Flores, A., \& Flores, R. (2013). Planificación estratégica por procesos para la empresa Satélite S.A.

Gavica Montoya, G. \& Garay Ramírez, A. 2012 Las empresas familiares: Por qué fracasan y su incidencia en la economía de la provincia de Los Ríos cantón Babahoyo. Un estudio de caso. Tesis de grado. Ecuador: Universidad Tecno-lógica de Babahoyo. Disponible en: http://repositorio.utb.edu.ec:8080/handle/123456789/654 de la.

Goyzueía, S. (2013). Modelo de gestión para las empresas familiares con perspectivas de crecimiento y sostenibilidad. Perspectivas, Vol. 16(31), 87-132.

Granbinsky, S. (2000). Mi tienda en el nuevo milenio (2da ed.). México: Del verbo emprende.

Hudson, M., Smart, A. y Bourne, M. (2001). Theory and practice in SME performance measurement systems. International Journal of Operations \& Production Management. 21(8), 1096-1115.

Ibrahim, N., Angelidis, J., \& Parsa, F. (2008). Strategic management of family businesses: Current findings and directions for the future research. Internacional Journal of Management, Vol. 25(1), 95-110.

Jaramillo, V. (1998). Reorganización y gestión de cambio en las Empresas Familiares: CasoM. Maritano S.A. Tesis de postgrado MBA. Valdivia: Universidad Austral de Chile.

Jaramillo, V. (2002). Las empresas familiares frente a la implementación de sistemas de control. FOROUM EMPRESARIAL, Vol. 7(2), 56-81.

Kaplan, R., \& Norton, D. (2011). Poniendo el Balanced Scorecard en acción. Harvard Business School, Vol. 89(11), 52-65.

Esta obra se comparte bajo la licencia Creative Common Atribución-No Comercial 4.0 International (CC BY-NC 4.0) 
Lozano, M. (2000). El protocolo en las empresas de propiedad familiar. Estudios Gerenciales, (74), 49-67

Molina, P., Botero, S., \& Montoya, J. (2016). Empresas de familia: conceptos y modelos para su análisis. Pensamiento y Gestión, Vol. (41), 116-149.

Montoya, C., \& Bárbaro, J. (2011). The Balanced Scorecard as a control system and its application as evaluation tool in the library environment. Revista Interoamericana de Bibliotecnología, 34(1), 35-47.

Peñaherrera, S., Ortiz, S., \& Herrera, J. (2017). Balanced Scorecard aplicado en empresas de calzado de la provincia de Tungurahua. Caso MELAN. Revista Publicando, Vol. 4(12).

Proaño, H., Gisbert, V., \& Pérez, E. (2017). Mejora continua enfocada a los problemas de empresas familiares. 3C Empresa, Investigación y Pensamiento crítico, 29-38.

Quejada, R., \& Ávila, J. (2016). Empresas Familiares: conceptos, teorías y estructuras. Revista de Escuela de Administración de Negocios, Vol. (81), 149-158.

Quintero, L., \& Osorio, L. (2018). Balanced Scorecard como herramienta para las empresas en estado de crisis. Revista CEA, Vol. 4(8), 75-94.

Rodríguez Díaz, R. 1997 La empresa familiar: Doce claves para el éxito. F. B. Sofesa. Madrid: Ed. Las Palmas. España

Ruiz, F., Saldaña, Y., Gaona, L., \& Jacobo, D. (2016). Concepto de familia en la cultura mexicana y su impacto en la empresa familiar. Revista Internacional Administración y Finanzas, Vol. 9(5), 15-25.

Santamaría, E., \& Pico, F. (2015). Sucesión de las empresas familiares: Análisis de los factores estratégicos que influyen en la dinámica familia-empresa. Revista Politécnica, Vol. 35(2).

Sánchez, F. (2010). El Balanced Scorecard como herramienta de gestión en las organizaciones del siglo XXI. Desarrollo y Gestión, Vol. 7(2), 123-132.

Sánchez, J., \& Araujo, P. (2016). Balanced Scorecard para emprendedores: Desde el modelo canvas al Cuadro de Mando Integral. Revista de la facultad de ciencias económicas de la Universidad Militar de Nueva Granada, Vol. XXIV (1), 37-47.

Scaramussa, S., Reisdorfer, V. \& Ribeiro, A. (2010). La contribución del Balanced Scorecard como instrumento de gestión estratégica en el apoyo a la gerencia. Revista Visión de Futuro, $13 \quad$ (1), Recuperado de http://www.fce.unam.edu.ar/revistacientifica/index.php?option=com_content\&view=artic le\&id=184\&Itemid=51

Suárez, F., \& Gonzáles, M. (2017). Análisis de la aplicación del Balanced Scorecard como herramienta de diagnóstico adaptada a microempresas. Revista de economía \& administración, Vol. 14(1), 99-110.

Torres, F. (2002). Balanced Scorecard centrado en lo ambiental. Cuadernos de Contabilidad. Vol. (14), 111.

Vallejo, M. (2005). Cuando definir es una necesidad. Una propuesta integrada y operativa del concepto de empresa familiar. Investigaciones Europeas, Vol. 11(3), 151-171.

Vélez, D., Holguín, H., De la Hoz, G., Durán, Y., \& Ayala, I. (2008). Dinámica de la empresa familiar Pyme: Estudio exploratorio en Colombia. Bogotá: Fundación para el desarrollo sostenible. Impreso por Fotolito Colombia Ltda. CTP Service.

Von Bergen, C. W. y Benco, D. C. (2004). A balanced scorecard for small business. Trabajo presentado en United States Association for Small Business and Entrepreneurship Conference. Texas.

Esta obra se comparte bajo la licencia Creative Common Atribución-No Comercial 4.0 International (CC BY-NC 4.0) 